\title{
SIMPLICIAL SCHREIER SYSTEMS AND THE COMMUTATOR SUBGROUP OF THE FREE GROUP ON THE CIRCLE
}

\author{
SABAH A. GHULLAM
}

\begin{abstract}
It is shown that the commutator subgroup of the free simplicial group on the circle has a simplicial Schreier system and is a free simplicial group on a pointed simplicial set.
\end{abstract}

Introduction. Subgroups of a free topological (simplicial) group need not be free topological (simplicial) [6], [9], [2], [3]. In [3] it is proved that the commutator subgroup $\left[F S^{n}, F S^{n}\right]$ of $F S^{n}(n>1)$ is not free topological. The homotopy theoretic methods used there were not helpful for $S^{1}$. In this paper, we use simplicial methods to construct a simplicial Schreier system for the subgroup $\left[F S^{1}, F S^{1}\right]$ in $F S^{1}$ and show that the free simplicial basis for $\left[F S^{1}, F S^{1}\right]$ is of the same homotopy type as $S^{2}$.

The referee has pointed out that the term "free simplicial group" has been used in two conflicting senses in the literature. Firstly, in the sense of Kan's and Whitehead's paper [10] where these groups are free in each dimension with generators which are stable under degeneracies but not necessarily under face maps. Secondly, in the sense of [2], and also [12], where these groups are free in each dimension with generators which are stable under both degeneracies and face maps. However, in our results the term "free simplicial group on a pointed simplicial set" is used in the second sense.

The author is greatly indebted to Dr. F. Clarke, who, as his research supervisor, gave invaluable assistance in the preparation of this paper. He also wishes to thank Professor R. Brown for his helpful comments.

1. Simplicial Schreier systems. Let $K$ be a pointed simplicial set, $F=F K$ be the free simplicial group on $K$ as defined by J. Milnor [11] (see also the appendix of [2]). The face and degeneracy operators $d_{i}, s_{i}$ are the homomorphic extensions of those in $K$. Let $K \rightarrow^{\eta} F$ be the inclusion and $H \subset F$ be a simplicial subgroup.

DEFINITION 1.1. A (two-sided) simplicial Schreier system for the cosets of a (normal) subgroup $H$ in $F$ is a subcomplex $S$ of $F$ such that for each $n \geqslant 0$, $S_{n}$ is a (two-sided) Schreier system (Hall [7]) for the cosets of $H_{n}$ in $F_{n}$. That is:

(i) each coset of $F_{n} / H_{n}$ contains exactly one element of $S_{n}$;

Received by the editors February 16, 1977 and, in revised form, May 2, 1977.

AMS (MOS) subject classifications (1970). Primary 22A99; Secondary 20E05, 55J10.

Key words and phrases. Free simplicial group, Schreier system. 
(ii) the identity $e_{n} \in S_{n}$;

(iii) if $x_{i_{1}}^{\varepsilon_{1}} \cdots x_{i_{m}}^{\varepsilon_{m}}$ is a reduced word in $S_{n}$, then $x_{i_{1}}^{\varepsilon_{1}} \cdots x_{i_{k}}^{\varepsilon_{k}}$ for $1 \leqslant k<m$ (and $x_{i_{h}}^{\varepsilon_{h}} \cdots x_{i_{k}}^{\varepsilon_{k}}$ for $1 \leqslant h \leqslant k \leqslant m$ ) is again in $S_{n}$.

Proposition 1.2. If $S$ is a simplicial Schreier system for the cosets of $H$ in $F$, then the map $\varphi: F / H \rightarrow F$, which sends a coset to its unique representative in $S$, is a simplicial section of the natural map $\pi: F \rightarrow F / H$.

Proof. For $x \in F_{n}$, let $\varphi_{n}\left(H_{n} x\right)=a \in S_{n}$. Then $H_{n} x \cap S_{n}=\{a\}$. Let $H_{n-1}\left(d_{i} x\right) \cap S_{n-1}=\{b\} \subset S_{n-1}$. Then $d_{i}\left(H_{n} x \cap S_{n}\right) \subset H_{n-1}\left(d_{i} x\right) \cap S_{n-1}$ $=\{b\}$, i.e. $d_{i} a=b$. Hence $d_{i} \varphi_{n}\left(H_{n} x\right)=\varphi_{n-1} d_{i}\left(H_{n} x\right)$. Similarly $s_{i} \varphi_{n}=\varphi_{n+1} s_{i}$. Clearly $\pi_{n} \varphi_{n}=$ id on $F_{n} / H_{n}$.

Theorem 1.3 (Simplicial Nielsen-Schreier Theorem). If $S=\{\varphi(H x)$ : $x \in F\}$ is a simplicial Schreier system for the cosets of $H$ in $F$, then there exists a subcomplex $L$ of $F$ such that $H=F L$.

Proof. Let $L_{n}=\left\{y x \varphi_{n}(H y x)^{-1}: y \in S_{n}, x \in K_{n}\right\}$. The usual NielsenSchreier Theorem (Hall [7]) shows that $L_{n} \backslash\left\{e_{n}\right\}$ is an algebraically free basis for $H_{n}$ for each $n \geqslant 0$.

Since $\varphi$ is simplicial, by Proposition 1.2, it follows that $L$ is stable under face and degeneracy operators, i.e. $L$ is a subcomplex of $F$ for which $H=F L$.

REMARK 1.4. Simplicial Schreier systems do not always exist, contrary to the algebraic case (Hall [7]) since simplicial subgroups of free simplicial groups are not always free simplicial ([6], [9], and [2]). For example, F. Clarke [3] has shown that the commutator subgroup $\left[F S^{n}, F S^{n}\right]$ of $F S^{n}$ is not free for $n>1$. In fact it is easy to see in this case that there is no simplicial section of the natural projection

$$
F S^{n} \stackrel{\pi}{\rightarrow} F S^{n} /\left[F S^{n}, F S^{n}\right] \cong A S^{n}
$$

where $A S^{n}$ is the free simplicial abelian group on the $n$-sphere, $S^{n}$. For this would imply that

$$
H^{*}\left(A S^{n} ; \mathbf{Z} / 2\right) \stackrel{\pi^{*}}{\rightarrow} H^{*}\left(F S^{n} ; \mathbf{Z} / 2\right)
$$

was a monomorphism. But $A S^{n}$ is a $K(\mathbf{Z}, n)([11$, Theorem 24.5] or [1, Theorem 5.12]), $F S^{n}$ has the homotopy type of $\Omega S^{n+1}$ [8], and $\pi^{*}$ maps the fundamental class $x \in H^{n}(K(\mathbf{Z}, n) ; \mathbf{Z} / 2)$ to the generator $y \in H^{n}\left(\Omega S^{n+1}\right.$; $Z / 2)$. Now $x^{2} \neq 0$ for $n>1$ but $y^{2}=0$, so $\pi^{*}\left(x^{2}\right)=0$.

Even if $H \subset F$ is a free simplicial subgroup, then $H$ may not have a simplicial Schreier system; this can be shown as follows: Let $I=[0,1]$ be the unit interval considered as a simplicial set with three nondegenerate simplices, $a$ in dimension 1 and $e, f$ in dimension 0 with $d_{0} a=e, d_{1} a=f ; e$ is the base point. Consider $H=F\{e, f\} \subset F I$, the free simplicial subgroup of $F I$ generated by $f$. Suppose $S \subset F I$ is a simplicial Schreier system for $H$ in $F I$; then, by Proposition 1.2, the map $\varphi$ is a simplicial section of the natural map $F I \rightarrow^{\pi} F I / H$. This would imply that $F I \cong H \times F I / H$ (as simplicial sets) 
and, hence, that $F I$ is not connected, which is a contradiction. Hence the existence of a simplicial Schreier system is not a necessary condition for freeness of a subgroup. This agrees with the case of a topological Schreier transversal [13].

On the other hand if $G$ is a simplicial group and $F G \rightarrow^{p} G$ is the unique homomorphism such that $p \eta=\mathrm{id}$, then clearly $\eta G \subset F G$ is a simplicial Schreier system for the kernel of $p$. In fact $\operatorname{ker} p \cong F(G \wedge G)$ [5].

We can construct a simplicial Schreier system for the commutator subgroup of $F K$ if $K$ can be simplicially ordered in the following way:

DEFINITION 1.5. Let $K$ be a pointed simplicial set with base point $e_{n}=s_{0}^{n} e$. Then $K$ is said to be simplicially ordered if there exists a total ordering on $K_{n} \backslash\left\{e_{n}\right\}$ such that $K_{n} \backslash\left\{e_{n}\right\} \rightarrow^{s_{i}} K_{n+1} \backslash\left\{e_{n+1}\right\}$ is order preserving and if $x<y$ in $K_{n} \backslash\left\{e_{n}\right\}$ implies $d_{i} x \leqslant d_{i} y$ unless $d_{i} x=e_{n-1}$ or $d_{i} y=e_{n-1}$.

In $\$ 2$ we will construct a simplicial ordering on the 1-sphere, $S^{1}$. Here we have the following results:

Proposition 1.6. If $\left\{K_{\lambda} ; \lambda \in \Lambda\right\}$ is a family of pointed simplicial sets each with a simplicial ordering, then $\bigvee_{\lambda \in \Lambda} K_{\lambda}$ has a simplicial ordering.

Proof. ( $\left.\bigvee_{\lambda \in \Lambda} K_{\lambda}\right)_{n} \backslash\left\{e_{n}\right\}=\prod_{\lambda \in \Lambda}\left(\left(K_{\lambda}\right)_{n} \backslash\left\{e_{n}\right\}\right)$. Totally order the indexing set $\Lambda$ and define the ordering on $\left(\bigvee_{\lambda \in \Lambda} K_{\lambda}\right)_{n} \backslash\left\{e_{n}\right\}$ as follows: $x<y$ if

(i) $x, y \in\left(K_{\lambda}\right)_{n} \backslash\left\{e_{n}\right\}$ and $x<y$ in the given ordering on $K_{\lambda}$; or

(ii) $x \in\left(K_{\lambda}\right)_{n} \backslash\left\{e_{n}\right\}, y \in\left(K_{\mu}\right)_{n} \backslash\left\{e_{n}\right\}$ and $\lambda<\mu$ in the ordering on $\Lambda$. It is easy to check that this defines a simplicial ordering on $\bigvee_{\lambda \in \Lambda} K_{\lambda}$.

THEOREM 1.7. If $K$ is a pointed simplicial set with a simplicial ordering, then there exists a simplicial Schreier system for the commutator subgroup [FK, FK] of $F K$.

Proof. Since $(A K)_{n} \cong(F K)_{n} /\left[(F K)_{n},(F K)_{n}\right]$ is the free abelian group on $K_{n}$, it follows that

$S_{n}=\left\{x_{1}^{k_{1}} x_{2}^{k_{2}} \cdots x_{m}^{k_{m}} \in(F K)_{n}: x_{1}<x_{2}<\cdots<x_{m}\right.$ in $\left.K_{n} \backslash\left\{e_{n}\right\}, k_{i} \in \mathbf{Z}\right\}$

is an algebraic, two-sided, Schreier system for $\left[(F K)_{n},(F K)_{n}\right]$ in $(F K)_{n}$. Now applying the face operator $d_{i}$ to $x_{1}^{k_{1}} x_{2}^{k_{2}} \cdots x_{m}^{k_{m}}$ we obtain $\left(d_{i} x_{1}\right)^{k_{1}}\left(d_{i} x_{2}\right)^{k_{2}} \cdots\left(d_{i} x_{m}\right)^{k_{m}}$ in $(F K)_{n-1}$. We can delete any $\left(d_{i} x_{j}\right)^{k_{j}}$ for which $d_{i} x_{j}=e_{n-1}$ to obtain an element of $S_{n-1}$, since $d_{i} x_{j} \leqslant d_{i} x_{k}$ if neither is the base point. Hence $d_{i} S_{n} \subset S_{n-1}$. Similarly $s_{i} S_{n} \subset S_{n+1}$, i.e. $S$ is a subcomplex of $F K$ and, hence, $S$ is a simplicial Schreier system.

Now Theorem 1.3 and 1.7 give

COROllaRY 1.8. If $K$ is a pointed simplicial set with a simplicial ordering, then the commutator subgroup of $F K$ is a free simplicial group on a pointed simplicial set.

2. A free simplicial basis for $\left[F S^{1}, F S^{1}\right]$. We will show how $S^{1}$ can be simplicially ordered, but before doing so we prove the following lemma: 
LEMMA 2.1. Let $K$ be a simplicial set with nondegenerate $a$ in $K_{1}$ and define $x_{j}=s_{n-1} \cdots \hat{s}_{j} \cdots s_{0} a$ in $K_{n}(0 \leqslant j \leqslant n-1)$ where $n$ denotes omission of the jth degeneracy. Then

(i)

$$
d_{i} x_{j}= \begin{cases}x_{j} & \text { if } i>j, j \neq n-1, \\ x_{j-1} & \text { if } i \leqslant j, j \neq 0\end{cases}
$$

while $d_{0} x_{0}=s_{0}^{n-1} d_{0} a$ and $d_{n} x_{n-1}=s_{0}^{n-1} d_{1} a$.

(ii)

$$
s_{i} x_{j}= \begin{cases}x_{j} & \text { if } i>j \\ x_{j+1} & \text { if } i \leqslant j\end{cases}
$$

Proof. If $j \leqslant n-2$, then $x_{j}=s_{n-1} x_{j}$ in $K_{n}$. Hence

$$
d_{i} x_{j}=d_{i} s_{n-1} x_{j}= \begin{cases}s_{n-2} d_{i} x_{j} & \text { if } i<n-1, \\ x_{j} & \text { if } i=n, n-1,\end{cases}
$$

while if $x_{n-1}=s_{n-2} x_{n-2}$ in $K_{n+1}$, then

$$
d_{i} x_{n-1}=d_{i} s_{n-2} x_{n-2}= \begin{cases}s_{n-3} d_{i} x_{n-2} & \text { if } i<n-2, \\ s_{n-2} d_{n-1} x_{n-2} & \text { if } i=n, \\ x_{n-2} & \text { if } i=n-2, n-2\end{cases}
$$

Clearly

and

$$
d_{0} x_{0}=d_{0}\left(s_{n-1} \cdots \hat{s}_{0} a\right)=s_{n-2} \cdots s_{0} d_{0} a=s_{0}^{n-1} d_{0} a
$$

$$
d_{n} x_{n-1}=d_{n}\left(\hat{s}_{n-1} \cdots s_{0} a\right)=s_{n-2} \cdots s_{0} d_{1} a=s_{0}^{n-1} d_{1} a .
$$

Hence (i) is proved. A similar argument can be used to show (ii).

THEOREM 2.2. If $S^{1}$ denotes the pointed simplicial set with two nondegenerate simplices, $e$ in dimension 0 and $a$ in dimension 1 , then $S^{1}$ has a simplicial ordering and, hence, $\left[F S^{1}, F S^{1}\right]$ is a free simplicial group on a pointed simplicial set.

Proof. The set of $n$-simplices in $S^{1}$ is

$$
\left(S^{1}\right)_{n}=\left\{e, x_{0}, x_{1}, \ldots, x_{n-1}\right\}
$$

where $e=s_{n-1} \cdots s_{0} e$ and $x_{j}=s_{n-1} \cdots \hat{s}_{j} \cdots s_{0} a(0 \leqslant j \leqslant n-1)$ [4, p. 110]. Define an ordering on $\left(S^{1}\right)_{n} \backslash\{e\}$ as follows:

$$
x_{i}<x_{j} \text { if } i<j, \quad i, j=0,1, \ldots, n-1 .
$$

Now Lemma 2.1 shows that this is a simplicial ordering and, hence, by Corollary 1.8, $\left[F S^{1}, F S^{1}\right]$ is a free simplicial group on a pointed simplical set.

Note. 1. A similar construction works for the unit interval and, hence, by Proposition 1.6, for any wedge of circles and intervals.

2. By means of the realization functor [2, Appendix], [11], corresponding 
statements can be obtained for free topological groups.

Now Theorem 2.2 shows that $\left[F S^{1}, F S^{1}\right] \cong F X^{\prime}$ for some pointed simplicial set $X$. We proceed to describe this set. It is clear that

$$
\begin{aligned}
S_{n}=\left\{x_{0}^{k_{0}} x_{1}^{k_{1}} \cdots x_{n-1}^{k_{n-1}} \in\left(F S^{1}\right)_{n}: x_{i}\right. & \in\left(S^{1}\right)_{n}, \\
k_{i} & \in \mathbf{Z}, 0 \leqslant i \leqslant n-1\}
\end{aligned}
$$

is the simplicial Schreier system for the subgroup $\left[F S^{1}, F S^{1}\right]$ in $F S^{1}$. In $\left(F S^{1}\right)_{n}$, write

$$
\begin{aligned}
w\left(j ; k_{0}, \ldots, k_{n-1}\right)=x_{0}^{k_{0}} \cdots x_{n-1}^{k_{n-1}} x_{j}\left(x_{0}^{k_{0}} \cdots x_{j}^{k_{j}+1} \cdots x_{n-1}^{k_{n-1}}\right)^{-1}, \\
0 \leqslant j \leqslant n-1 .
\end{aligned}
$$

Then we have

Proposition 2.3. (i) $w\left(j ; k_{0}, \ldots, k_{n-1}\right) \neq e$ if and only if there exists $k_{i} \neq 0$ for some $i>j$.

(ii)

$$
\begin{aligned}
& d_{i} w\left(j ; k_{0}, \ldots, k_{n-1}\right) \\
& = \begin{cases}w\left(j ; k_{0}, \ldots, k_{i}+k_{i+1}, \ldots, k_{n-1}\right) & \text { if } i>j \text { and } i \neq n, \\
w\left(j-1 ; k_{0}, \ldots, k_{i}+k_{i+1}, \ldots, k_{n-1}\right) & \text { if } i \leqslant j \text { and } i \neq 0, n,\end{cases} \\
& s_{i} w\left(j ; k_{0}, \ldots, k_{n-1}\right)= \begin{cases}w\left(j ; k_{0}, \ldots, k_{i-1}, 0, k_{i}, \ldots, k_{n-1}\right) & \text { if } i>j, \\
w\left(j+1 ; k_{0}, \ldots, k_{i-1}, 0, k_{i}, \ldots, k_{n-1}\right) & \text { if } i \leqslant j,\end{cases}
\end{aligned}
$$

while

$$
d_{0} w\left(j ; k_{0}, \ldots, k_{n-1}\right)=\left\{\begin{array}{l}
w\left(j-1 ; k_{1}, \ldots, k_{n-1}\right) \quad \text { if } j \neq 0 \\
e \text { if } j=0
\end{array}\right.
$$

and

$$
d_{n} w\left(j ; k_{0}, \ldots, k_{n-1}\right)=w\left(j ; k_{0}, \ldots, k_{n-2}\right) .
$$

Proof. Follows easily from Lemma 2.1.

The simplicial Nielsen-Schreier Theorem, Theorem 1.3 and Proposition 2.3 show that the set

$$
\begin{aligned}
X_{n}=\left\{w\left(j ; k_{0}, \ldots, k_{n-1}\right) \in\left(F S^{1}\right)_{n}: \exists k_{i} \neq 0\right. \\
\left.\quad \text { for some } i>j, k_{i} \in \mathbf{Z}, 0 \leqslant j \leqslant n-2\right\}
\end{aligned}
$$

is a free basis for $\left[\left(F S^{1}\right)_{n},\left(F S^{1}\right)_{n}\right]$ for each $n \geqslant 0$ so that $\left[F S^{1}, F S^{1}\right]$ is isomorphic to the simplicial group $F X$. We claim that $X$ is of the same homotopy type as $S^{1} \wedge A S^{1}$ and, hence, of $S^{2}$, since $A S^{1}$ is a $K(\mathrm{Z}, 1)$. To prove this we define $p: S^{1} \wedge A S^{1} \rightarrow X$ as follows:

$$
p: x_{j} \wedge x_{0}^{k_{0}} \ldots x_{n-1}^{k_{n-1}} \mapsto w\left(j ; k_{0}, \ldots, k_{n-1}\right)
$$

where $x_{j} \in\left(S^{1}\right)_{n}, 0 \leqslant j \leqslant n-1$, and $k_{i} \in \mathbf{Z}$. It is easy to check that $p$ is a simplicial map which identifies $X$ with $S^{1} \wedge A S^{1} / T$ where $T=p^{-1}(e)$, that is 


$$
T_{n}=\left\{x_{j} \wedge x_{0}^{k_{0}} \cdots x_{j}^{k_{j}}: x_{j} \in\left(S^{1}\right)_{n}, k_{i} \in \mathbf{Z}, 0 \leqslant j \leqslant n-1\right\} .
$$

Proposition 2.4. $T$ is contractible.

Proof. Let $h_{s}: T_{n} \rightarrow T_{n+1}, 0 \leqslant s \leqslant n$, be defined by

$$
h_{s}\left(x_{j} \wedge x_{0}^{k_{0}} \cdots x_{j}^{k_{j}}\right)=\left\{\begin{array}{l}
x_{s} \wedge x_{0}^{k_{0}} \cdots x_{j}^{k_{j}} \text { if } s>j, \\
x_{j+1} \wedge x_{0}^{k_{0}} \cdots x_{s-1}^{k_{s-1}} x_{s+1}^{k_{s}} x_{s+2}^{k_{s+1}} \cdots x_{j+1}^{k_{j}} \quad \text { if } s \leqslant j .
\end{array}\right.
$$

It can be checked easily, but tediously [5], that $h$ is a contracting homotopy for $T$, as defined by the relations (i)-(iii) of [11, Definition 5.1].

Now it follows from the cofibration $T \hookrightarrow^{i} S^{1} \wedge A S^{1} \hookrightarrow^{p} X$ that $p$ is a homotopy equivalence.

\section{REFERENCES} 545-565.

1. R. Brown, Cohomology with chains as coefficients, Proc. London Math. Soc. (3) 14 (1964),

2. __ Some nonprojective subgroups of free topological groups, Proc. Amer. Math. Soc. 52 (1975), 433-438.

3. F. W. Clarke, The commutator subgroup of a free topological group need not be projective, Proc. Amer. Math. Soc. 58 (1976), 354-356.

4. E. B. Curtis, Simplicial homotopy theory, Advances in Math. 6 (1971), 107-209.

5. S. A. Ghullam, Univ. of Wales thesis, (in preparation).

6. M. I. Graev, Free topological groups, Izv. Akad. Nauk SSSR Ser. Mat. 12 (1948), 279-324; English transl., Amer. Math. Soc. Transl. (1) 8 (1962), 305-364.

7. M. Hall, Jr., The theory of groups, Macmillan, New York, 1962.

8. J. P. Hardy and D. Puppe, Classifying spaces and universal categories (to appear).

9. D. C. Hunt and S. A. Morris, Free subgroups of free topological groups, Proc. Second Internat. Conf. Theory of Groups, Lecture Notes in Math., vol. 372, Springer-Verlag, Berlin and New York, 1974, pp. 377-387.

10. D. M. Kan and G. W. Whitehead, Orientability and Poincare duality in general homology theories, Topology, 3 (1965), 231-270.

11. J. P. May, Simplicial objects in algebraic topology, Van Nostrand, Princeton, N. J., 1967.

12. J. W. Milnor, The construction FK, A student's guide to algebraic topology by J. F. Adams, London Math. Soc. Lecture Notes 4 (1972).

13. P. Nickolas, A Schreier theorem for free topological groups, Bull. Austral. Math. Soc. 13 (1975), 121-127.

Department of Pure Mathematics, University College of Swansea, Wales, Untted KINGDOM

Current address: Faculty of Economics, Garyounis University, Benghazi, Libya 